10. Dong L, Wang Y, Zuo W, et al. Unusual intravascular leiomyomatosis arising from the pulmonary artery. Eur Heart J Cardiovasc Imaging. 2016;17:844.

Antonio Figueredo ${ }^{\mathrm{a}}$, Wilmer Rivero ${ }^{\mathrm{b}}$, Adriana Murcia ${ }^{\mathrm{a}}$, Ady Perroni ${ }^{a}$

y Mercedes Botia ${ }^{\mathrm{a}, *}$

a Departamento de Cirugía Cardiovascular, Fundación Cardiovascular de Colombia, Floridablanca, Santander, Colombia

${ }^{\mathrm{b}}$ Departamento de Urología, Fundación Cardiovascular de Colombia, Floridablanca, Santander, Colombia
* Autor para correspondencia. Calle 200 N. 13-200 Park 200 Torre 2 Apartamento 1104. Bucaramanga, Colombia. Teléfono: +3188338629.

Correo electrónico: mariabotia@fcv.org (M. Botia).

https://doi.org/10.1016/j.acmx.2018.02.010 1405-9940/

(c) 2018 Instituto Nacional de Cardiología Ignacio Chávez. Publicado por Masson Doyma México S.A. Este es un artículo Open Access bajo la licencia CC BY-NC-ND (http: / / creativecommons.org/ licenses/by-nc-nd/4.0/).

\section{Infiltración miocárdica por tumor, simulando isquemia miocárdica en electrocardiograma}

\section{Myocardial tumour infiltration mimicking myocardial ischaemia in electrocardiogram}

\section{Introducción}

Las metástasis por tumores malignos comúnmente no envuelven al corazón, sin embargo, cuando ocurren pueden causar daño del miocardio o pericardio, produciendo cambios en el electrocardiograma (ECG) en el segmento ST o la onda $T^{1}$. Estas metástasis también pueden inducir arritmias, bradicardia, fibrilación auricular, extrasístoles supraventriculares o ventriculares, trastornos de conducción, bloqueos auriculoventriculares. La invasión o compresión de alguna arteria coronaria principal ha sido reportada, pero es rara ${ }^{2}$. Los tumores cardiacos primarios son raros, y se encuentran hasta en $0.03 \%$ de autopsias. Los tumores cardiacos secundarios o metastásicos comparativamente son más frecuentes, con incidencia hasta 100 veces mayor que los primarios ${ }^{3}$.

\section{Caso clínico}

Paciente mujer de 69 años quien ingresó en la Unidad de Cuidados Coronarios del Hospital Universitario de Los Andes por haber presentado súbitamente dolor torácico opresivo, de fuerte intensidad, irradiado a brazo izquierdo, se mantuvo más de $20 \mathrm{~min}$, y se asoció a disnea. Al examen físico de ingreso presentaba frecuencia cardiaca de 93 lpm, frecuencia respiratoria de $26 \mathrm{rpm}$, presión arterial $110 / 80 \mathrm{mmHg}$. A la auscultación cardiaca presentaba soplo eyectivo en foco pulmonar grado $\mathrm{III} / \mathrm{vl}$, irradiado a foco aórtico accesorio, eyectivo, grado III/ıv. En la auscultación pulmonar se encontraron crepitantes finos y gruesos basales bilaterales. En ECG se observó elevación del punto $\mathrm{J}$ y ondas $\mathrm{T}$ negativas, simétricas de V1 a V4 y discreta elevación de ST en cara inferior (fig. 1). Tiene como antecedente imagen quística gigante en riñón derecho, a la cual realizaron en diversas oportunidades drenaje percutáneo, pero no realizaron biopsia ni indicaron otro tratamiento. También refirió infecciones respiratorias a repetición, con último episodio dos semanas antes de la hospitalización. En la radiografía de tórax se observaron imágenes redondeadas de aspecto algodonoso con distribución difusa en ambos campos pulmonares. Se solicitaron enzimas cardiacas y se encontraron dentro de límites normales en tomas seriadas; dímero $D$ resultó negativo. Se realizó ecocardiograma transtorácico donde se observó el ventrículo izquierdo (vı) no dilatado con patrón geométrico normal y fracción de eyección preservada, sin trastornos de cinesia regional; el ventrículo derecho (VD) no dilatado con función sistólica preservada, con imagen ecorrefringente adherida al ápex del VD, de $36 \times 20 \mathrm{~mm}$ aproximadamente, que se extendía hasta el tracto de salida del VD; presión sistólica de la arteria pulmonar normal. Ante tales hallazgos, se solicitó resonancia magnética de tórax y abdomen, con contraste (fig. 2), que reportó lesión ocupante de espacio (LOE) heterogénea en cavidad del VD, de gran tamaño $41 \times 79 \times 24 \mathrm{~mm}$, que infiltraba las paredes y ocasionaba disrupción del pericardio visceral, con bordes irregulares; generando obstrucción dinámica del tracto de salida del VD. Realce tardío heterogéneo con áreas de baja señal de intensidad en relación con necrosis tumoral y hemorragia. Se planteó sarcoma indiferenciado o lesión metastásica. Riñón derecho con pérdida de la morfología, lesión sólida heterogénea de bordes mal definidos, $6.9 \times 5.8 \mathrm{~cm}$. En ambos pulmones, imágenes nodulares múltiples, con diámetros entre 0.5 y $2 \mathrm{~cm}$, de distribución aleatoria, algunas con contacto pleural y con realce significativo de todas las lesiones.

Se descartó cardiopatía isquémica ante enzimas cardiacas negativas y ausencia de trastornos de cinesia en ecocardiograma, y se planteó que los cambios electrocardiográficos eran debidos a infiltración tumoral en el VD, y que esta era de origen metastásico, secundarias a LOE renal. La paciente egresó con valoración ambulatoria planificada por servicio de oncología. Dos meses después del alta, ingresó en el área de urgencias por presentar trombosis venosa profunda en ambos miembros inferiores, presentó deterioro progresivo del estado general, y falleció durante la hospitalización.

\section{Discusión}

Las metástasis cardiacas de otros tumores primarios siguen siendo poco comunes, a pesar de que ocurren más frecuentemente que los tumores cardiacos primarios ${ }^{1}$. Carcinoma de células renales, sarcomas y cáncer de pulmón 

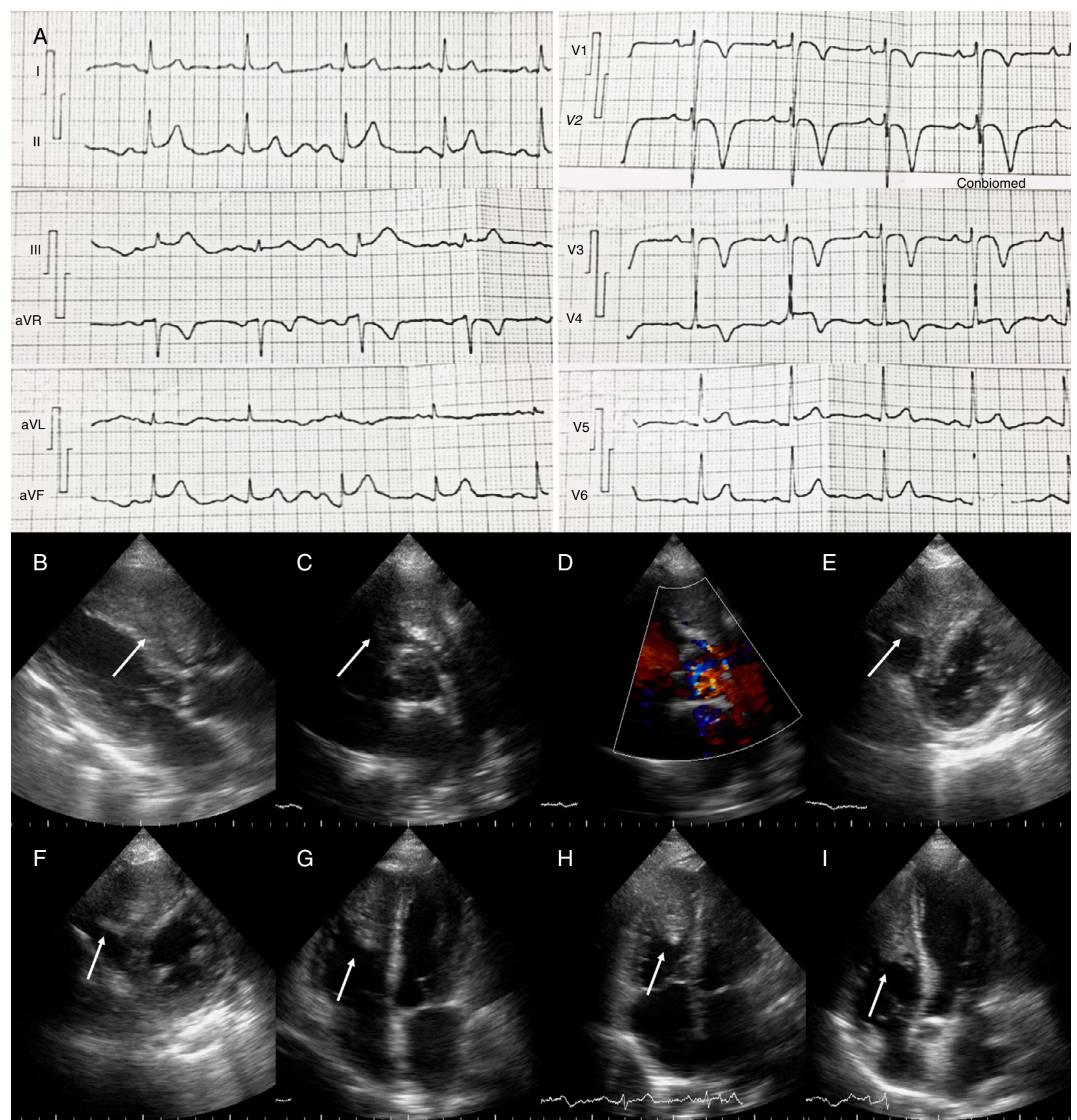

Figura 1 A) Electrocardiograma del paciente donde se observa elevación del punto J y ondas T negativas, simétricas de V1 a V4 y discreta elevación de ST en cara inferior (II, III y AVF). B-ı) Ecocardiograma transtorácico. B) Eje paraesternal largo, se observa imagen ecorrefringente dentro de gran parte de la cavidad del VD (flecha). C y D) Eje paraesternal corto al nivel de los grandes vasos, tumor en el tracto de salida del VD (flecha), con obstrucción dinámica vista por Doppler color D). E y F) Eje paraesternal corto al nivel de la valvular mitral y músculos papilares respectivamente. Se puede ver LOE en más del 50\% del VD (flechas). G y H) Vista de 4 cámaras y 4 cámaras modificado, donde se observa tumor intracavitario adherido al ápex y pared lateral del VD, y al septum interventricular (flecha). ı) Vista de 5 cámaras. Se observa la extensión del tumor dentro del VD y el septum interventricular (flecha).

han sido los tipos de cáncer más prevalentes asociados a metástasis cardiacas ${ }^{4}$. Las neoplasias hematológicas, ováricas, gástricas y pancreáticas también tienen altas tasas de dichas metástasis. El pericardio es el sitio mayormente involucrado, seguido de la afectación epicárdica y miocárdica.
Aunque cualquier tipo de tumor puede afectar el corazón, la probabilidad de esto es una función de consideraciones anatómicas, estadio de la enfermedad y la biología individual del tumor y del paciente ${ }^{5}$. Las metástasis endocárdicas e intracavitarias son raras, y cuando ocurren pueden pro- 


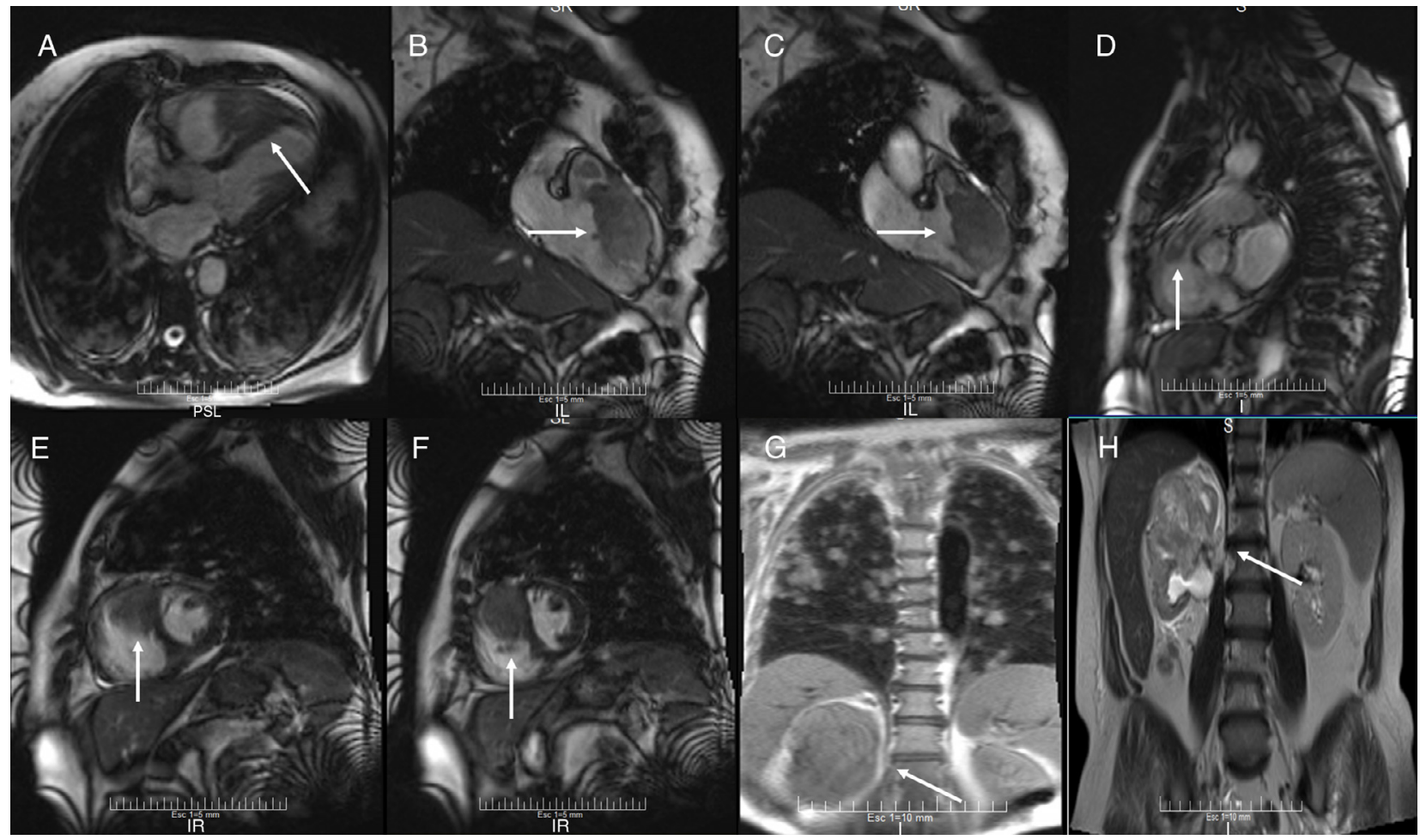

Figura 2 Resonancia magnética. A) Vista anatómica de 2 cámaras, en la que se ve tumor desde el ápex del VD hasta la parte media del VD, adherido también a la pared libre del VD y al septum interventricular (flecha). B y C) Vista lateral del VD en diástole y sístole respectivamente. En esta se confirma la extensión del tumor al tracto de salida del VD, así como también la obstrucción dinámica durante la sístole (flecha). D) Vista lateral del tracto de salida del VD. Se observa tumor extendido al origen de la arteria pulmonar (flecha). E y F) Vista de eje corto, donde se puede apreciar el tumor intracavitario y su extensión a través de la pared del VD hasta el pericardio (flecha). G y H) Resonancia magnética de tórax y abdomen. Se observa LOE de gran tamaño en riñón derecho e imágenes metastásicas en ambos pulmones (flecha).

ducir consecuencias severas como embolismos sistémicos o pulmonares, obstrucción del flujo de entrada o del tracto de salida del ventrículo afectado. En esta paciente se observó infiltración en ápex del VD y del tracto de salida del VD con obstrucción dinámica del mismo, y disrupción del pericardio visceral.

Los tumores pueden alcanzar el corazón mediante 4 vías: diseminación hematógena, diseminación linfática, extensión transvenosa y extensión directa. Ciertos tumores como el carcinoma de células renales y el carcinoma hepatocelular pueden extenderse dentro de la vena cava inferior y crecer dentro de la aurícula derecha (extensión transvenosa) ${ }^{5}$. En nuestra paciente, debido a sus antecedentes, se planteó que el tumor primario era del sistema renal, el cual hizo metástasis en pulmones y en VD. El estado hipercoagulable relacionado con el cáncer también puede causar la formación de trombos in situ ${ }^{1}$.

La mayoría de las metástasis cardiacas no se manifiestan clínicamente y se diagnostican posmortem; cuando se manifiestan dependen de su localización y pueden incluir disnea, palpitaciones, fibrilación o flutter auricular, edema en miembros inferiores, dolor torácico, derrame pericárdico y taponamiento cardiaco ${ }^{5}$. En este caso, la paciente presentó aparición súbita de dolor torácico opresivo, y se asoció a disnea.
Aun cuando no hay afectación de arterias coronarias, las metástasis en miocardio y pericardio podrían simular un síndrome coronario agudo, inclusive con elevación de biomarcadores cardiacos y alteraciones del segmento ST y la onda $\mathrm{T}$ en el $\mathrm{ECG}^{1,5}$. Otros mecanismos sugeridos para estos cambios son: lesión miocárdica continua que evita formación de nueva membrana celular, estiramiento de fibras musculares adyacentes, reacción inflamatoria, cambio en el gradiente $\mathrm{Na}-\mathrm{K}$ transmembrana y transferencia de $\mathrm{K}$ del tejido dañado al miocardio adyacente produciendo diferencias en los potenciales eléctricos ${ }^{6}$. En este caso había alteraciones del ST en cara inferior y del punto $\mathrm{J}$ y onda T en cara anteroseptal, sin embargo, las enzimas cardiacas normales, y dímero $D$ negativo, orientó a que los cambios en ECG no estaban asociados a infarto agudo de miocardio o embolismo pulmonar, pero podrían estar relacionados con la afectación del ápex y del septum interventricular a pesar de que el tumor se infiltró principalmente en VD.

La principal herramienta de detección es el ecocardiograma transtorácico, para establecer diagnóstico, localización y extensión de masas cardiacas. En algunos casos se debe usar el ecocardiograma transesofágico para mejor evaluación del patrón morfológico. La resonancia magnética cardiaca es excelente herramienta para diagnosticar, ubicar y medir $\mathrm{LOE}^{6}$. En este caso se realizó RM de 
tórax y abdomen para determinar la morfología del LOE renal y presencia de otras lesiones metástasis en ambos pulmones.

Aunque el diagnóstico clínico de metástasis cardiacas puede ser hecho mediante técnicas de imagen, la histología del tejido sigue siendo el método definitivo para diferenciar las masas neoplásicas de las no neoplásicas y para planear la terapia definitiva o paliativa. Para la obtención de muestras para biopsia se puede realizar toracotomía con biopsia abierta, biopsia endomiocárdica, y estudio de líquido pericárdico. En los casos de derrame pericárdico debido a malignidad, se pueden identificar células malignas cuando se realiza citología del líquido obtenido mediante pericardiocentesis; este resultado tiene alta correlación con el diagnóstico histológico ${ }^{5}$. Cuando se reseca una masa cardiaca, debe documentarse a través de evaluación exhaustiva. Deben describirse las características generales del tumor: color, consistencia, superficie (lisa, áspera o vellosa), tipo de crecimiento, presencia de pedículo. Además, se debe medir y pesar, describir la presencia de epicardio, endocardio o miocárdico y de cualquier estructura normal que se identifique (válvula, septum interauricular, etc.). También se debe documentar la distancia del tumor desde los márgenes. Las secciones cortadas se deben inspeccionar para detectar hemorragias, necrosis, calcificaciones o degeneración quística ${ }^{7}$.

La biopsia endomiocárdica es una herramienta muy útil para el diagnóstico preoperatorio de masas intracardiacas, principalmente las que se encuentran en cavidades derecha y que muestran un patrón de crecimiento infiltrante u obstructivo, para realizar diagnóstico diferencial de sarcomas, linfomas y tumores metastásicos, y en las masas cardiacas que no se pueden resecar. Durante la histología se realiza diferenciación de las masas entre neoplásicas y no neoplásicas, benignas y malignas, y se clasifican los subtipos de neoplasias, ya que el comportamiento biológico del tumor podría favorecer la formación de trombos, aumento en secreción de catecolaminas o mediadores de inflamación ${ }^{8}$.

En la inmunohistoquímica, el tipo de anticuerpos que se pueden utilizar depende del tipo de tumor. Un panel inicial útil sugerido incluye vimentina, antígeno relacionado con factor VIII (factor von Willebrand humano), CD31, CD34, antígeno de membrana epitelial, citoqueratinas de amplio espectro, proteína S-100, actina de músculo liso, desmina, miogenina, molécula HMB-45, CD45, CD20, y CD3. En los casos donde se aplica la biología molecular, se realiza comúnmente hibridación fluorescente in situ y las pruebas de $\mathrm{PCR}^{8}$. En nuestra paciente no se pudo obtener muestra de las masas observadas.

\section{Bibliografía}

1. Lu DY, Yu WC, Chen CK, et al. Tumor invasion of myocardium presented with acute coronary syndrome. Acta Cardiol Sin. 2015;31:257-60.

2. Suga T, Akuzawa N, Hatori T, et al. ST segment elevation in secondary cardiac cancer: a case report and review of the literature. Int J Clin Exp Med. 2015;8:7719-27.

3. Yamac AH, Insanic D, Bockmeyer C. Cardiac metastasis from a urothelial cell carcinoma: a commented case report. Cardiovasc Pathol. 2014;23:178-80.

4. Yusuf SW, Bathina JD, Qureshi S, et al. Cardiac tumors in a tertiary care cancer hospital: clinical features, echocardiographic findings, treatment and outcomes. Heart Int. 2012;7:20-6.

5. Goldberg AD, Blankstein R, Padera RF. Tumors metastatic to the heart. Circulation. 2013;128:1790-4.

6. Di Michele S, Mirabelli F, Galzerano D, et al. An unusual myocardial infarction. Echo Res Pract. 2014:K9-12.

7. Stone JR, Basso C, Baandrup UT, et al. Recommendations for processing cardiovascular surgical pathology specimens: a consensus statement from the Standards and Definitions Committee of the Society for Cardiovascular Pathology and the Association for European Cardiovascular Pathology. Cardiovasc Pathol. 2012;21: 2-16.

8. Leone $\mathrm{O}$, Veinot JP, Angelini A, et al. Consensus statement on endomyocardial biopsy from the Association for European Cardiovascular Pathology and the Society for Cardiovascular Pathology. Cardiovasc Pathol. 2012;21:245-74.

Carlos Eduardo Vergara-Uzcategui*, Nelsy González y José Hipólito Donís

Servicio de Cardiología, Instituto de Investigaciones Cardiovasculares, Hospital Universitario de Los Andes, Universidad de Los Andes, Mérida, Venezuela

* Autor para correspondencia. Dirección: Avenida 16 de Septiembre, Hospital Universitario de Los Andes. Nivel Emergencia. Mérida, Venezuela. Código Postal 05101. Correo electrónico: carting1@gmail.com

(C.E. Vergara-Uzcategui).

https://doi.org/10.1016/j.acmx.2017.11.007

1405-9940/

๑ 2017 Instituto Nacional de Cardiología Ignacio Chávez. Publicado por Masson Doyma México S.A. Este es un artículo Open Access bajo la licencia CC BY-NC-ND (http: / / creativecommons.org/ licenses/by-nc-nd/4.0/). 